\title{
Minimally Intersecting Set Partitions of Type $B$
}

\author{
William Y.C. Chen and David G.L. Wang \\ Center for Combinatorics, LPMC-TJKLC \\ Nankai University, Tianjin, P.R. China \\ chen@nankai.edu.cn, wgl@cfc.nankai.edu.cn
}

Submitted: Oct 6, 2009; Accepted: Jan 25, 2010; Published: Jan 31, 2010

Mathematics Subject Classification: 05A15, 05A18

\begin{abstract}
Motivated by Pittel's study of minimally intersecting set partitions, we investigate minimally intersecting set partitions of type $B$. Our main result is a formula for the number of minimally intersecting $r$-tuples of $B_{n}$-partitions. As a consequence, it implies the formula of Benoumhani for the Dowling number in analogy to Dobiński's formula.
\end{abstract}

\section{Introduction}

This paper is primarily concerned with the meet structure of the lattice of type $B_{n}$ partitions of the set $\{ \pm 1, \pm 2, \ldots, \pm n\}$. The lattice of type $B_{n}$ set partitions has been studied by Reiner [8]. It can be regarded as a representation of the intersection lattice of the type $B$ Coxeter arrangements, see Björner and Wachs [3], Björner and Brenti [2] and Humphreys [6].

A set partition of type $B_{n}$ is a partition $\pi$ of the set $\{ \pm 1, \pm 2, \ldots, \pm n\}$ into blocks satisfying the following conditions:

(i) For any block $B$ of $\pi$, its opposite $-B$ obtained by negating all elements of $B$ is also a block of $\pi$;

(ii) There is at most one zero-block, which is defined to be a block $B$ such that $B=-B$. We call $\pm B$ a block pair of $\pi$ if $B$ is a non-zero-block of $\pi$. For example,

$$
\pi_{1}=\{\{ \pm 1, \pm 2, \pm 5, \pm 8, \pm 12\}, \pm\{3,11\}, \pm\{4,-7,9,10\}, \pm\{6\}\}
$$

is a $B_{12}$-partition consisting of 3 block pairs and the zero-block $\{ \pm 1, \pm 2, \pm 5, \pm 8, \pm 12\}$.

Our main result is a formula for the number of $r$-tuples of minimally intersecting $B_{n^{-}}$ partitions. We have used similar ideas in Pittel [7], but the variable setting for type $B$ does not seem to be a straightforward generalization. 
Let us give a precise formulation of Pittel's results. Let $\Pi_{n}$ be the lattice of partitions of $[n]=\{1,2, \ldots, n\}$. The minimum element in $\Pi_{n}$ is

$$
\hat{0}=\{\{1\},\{2\}, \ldots,\{n\}\} .
$$

The partitions $\pi_{1}, \pi_{2}, \ldots, \pi_{r}$ are said to intersect minimally if

$$
\pi_{1} \wedge \pi_{2} \wedge \cdots \wedge \pi_{r}=\hat{0} .
$$

Let $\pi$ be a partition of the set $[n]$, and let $i_{1}, \ldots, i_{k}$ be the sizes of the blocks of $\pi$ listed in any order. Given $l>1$, the number $N(\pi, l)$ of partitions with exactly $l$ blocks that minimally intersect $\pi$ equals

$$
N(\pi, l)=\frac{\mathbf{i} !}{l !}\left[\mathbf{x}^{\mathrm{i}}\right]\left(\prod_{\alpha \in[k]}\left(1+x_{\alpha}\right)-1\right)^{l},
$$

where

$$
\mathbf{i} !=\prod_{\alpha \in[k]} i_{\alpha} !
$$

and $\left[\mathrm{x}^{\mathrm{i}}\right]$ stands for the coefficient of $\mathrm{x}^{\mathrm{i}}$ in the power series expansion. As pointed out by Pittel, the expression (1.1) reduces to Dobiński's formula. In other words, setting $\pi=\hat{0}$ one obtains

$$
B_{n}=e^{-1} \sum_{k \geqslant 0} \frac{k^{n}}{k}
$$

where $B_{n}$ denotes the Bell number. Moreover, in view of (1.1), Pittel deduced that the number $N(\pi)$ of partitions that minimally intersect $\pi$ equals

$$
N(\pi)=\mathbf{i} !\left[\mathbf{x}^{\mathrm{i}}\right] \exp \left(\prod_{\alpha \in[k]}\left(1+x_{\alpha}\right)-1\right) .
$$

Pittel also obtained the number $N_{2}(k)$ of ordered pairs $\left(\pi, \pi^{\prime}\right)$ of minimally intersecting partitions such that $\pi$ consists of exactly $k$ blocks, that is,

$$
N_{2}(k)=e^{-1} \frac{n !}{k !}\left[x^{n}\right] \sum_{l \geqslant 0} \frac{1}{l !}\left[(1+x)^{l}-1\right]^{k} .
$$

Using the above formula, he further derived the following expression for the number $N_{2 n}$ of ordered pairs of minimally intersecting partitions

$$
N_{n, 2}=e^{-2} \sum_{k, l \geqslant 0} \frac{(k l)_{n}}{k ! l !}
$$


where $(m)_{n}=m(m-1) \cdots(m-n+1)$ denotes the falling factorial. By the same method, Pittel generalized (1.5) and showed that the number $N_{n, r}$ of $r$-tuples $(r \geqslant 2)$ of minimally intersecting partitions equals

$$
N_{n, r}=\frac{1}{e^{r}} \sum_{k_{1}, \ldots, k_{r} \geqslant 0} \frac{\left(k_{1} k_{2} \cdots k_{r}\right)_{n}}{k_{1} ! k_{2} ! \cdots k_{r} !} .
$$

Canfield [4] found a formula connecting the generating functions of $N_{n, r}$ and the $r$-th power of Bell numbers.

The set of partitions of type $B$ on $\{ \pm 1, \pm 2, \ldots, \pm n\}$ forms a lattice under refinement, denoted $\Pi_{n}^{B}$, with the minimal element

$$
\hat{0}^{B}=\{ \pm\{1\}, \pm\{2\}, \ldots, \pm\{n\}\} .
$$

The $B_{n}$-partitions $\pi_{1}, \pi_{2}, \ldots, \pi_{r}$ are said to be minimally intersecting if

$$
\pi_{1} \wedge \pi_{2} \wedge \cdots \wedge \pi_{r}=\hat{0}^{B}
$$

We shall study the meet structure of $\Pi_{n}^{B}$ in analogy with Pittel's formulas. Our main result is the following theorem.

Theorem 1.1 Let $r \geqslant 2$. The number of minimally intersecting $r$-tuples $\left(\pi_{1}, \pi_{2}, \ldots, \pi_{r}\right)$ of $B_{n}$-partitions equals

$$
N_{n, r}^{B}=\frac{2^{n}}{e^{r / 2}} \sum_{k_{1}, \ldots, k_{r} \geqslant 0} \frac{\left(f_{r}\right)_{n}}{\left(2 k_{1}\right) ! !\left(2 k_{2}\right) ! ! \cdots\left(2 k_{r}\right) ! !},
$$

where

$$
f_{r}=\frac{1}{2}\left(\prod_{t \in[r]}\left(2 k_{t}+1\right)-1\right)
$$

The proof of the above formula leads to a formula of Benoumhani [1] for the number of $B_{n}$-partitions, called the Dowling number [5]. This paper is organized as follows. In the next section, we derive type $B$ analogues of the formulas from (1.1) to (1.6) and we give a proof of Theorem 1.1. In Section 3, we shall consider the corresponding problems with respect to $B_{n}$-partitions without zero-block.

\section{Minimally intersecting $B_{n}$-partitions}

The main objective of this section is to derive a formula for the number of minimally intersecting $r$-tuples of $B_{n}$-partitions. If $\pi \in \Pi_{n}^{B}$ has a zero-block $Z=\left\{ \pm r_{1}, \pm r_{2}, \ldots, \pm r_{k}\right\}$, we say that $Z$ is of half-size $k$. Let $\mathbf{j}=\left(j_{1}, j_{2}, \ldots, j_{k}\right)$ be a composition of $n$. Let $\pi$ be a $B_{n}$-partition consisting of $k$ block pairs and a zero-block of half-size $i_{0}$. We often assume that the block pairs of $\pi$ are ordered subject to certain convention for the purpose of 
enumeration. We say that $\pi$ is of type $\left(i_{0} ; \mathbf{j}\right)$ if the block pairs of $\pi$ are ordered such that the $i$-th block pair is of length $j_{i}$.

We first consider the problem of counting the number of $B_{n}$-partitions with $l$ block pairs which minimally intersect a given $B_{n}$-partition.

Theorem 2.1 Let $\pi$ be a $B_{n}$-partition consisting of a zero-block of half-size $i_{0}$ (allowing $\left.i_{0}=0\right)$ and $k$ block pairs of sizes $i_{1}, i_{2}, \ldots, i_{k}(k \geqslant 1)$ listed in any order. For any $l \geqslant 1$, the number of $B_{n}$-partitions $\pi^{\prime}$ containing exactly $l$ block pairs that minimally intersect $\pi$ equals

$$
N^{B}(\pi ; l)=\frac{\mathbf{i} !}{\left(2 l-2 i_{0}\right) ! !} \sum_{\mathbf{i}^{\prime}}\left[\mathbf{x}^{\mathbf{i}^{\prime}}\right]\left(\prod_{\alpha \in[k]}\left(1+x_{\alpha}\right)^{2}-1\right)^{l-i_{0}} \prod_{\alpha \in[k]}\left(1+x_{\alpha}\right)^{2 i_{0}}
$$

where $\mathbf{i}^{\prime}$ ranges over all vectors $\left(i_{1}^{\prime}, i_{2}^{\prime}, \ldots, i_{k}^{\prime}\right)$ such that $i_{\alpha}^{\prime} \in\left\{i_{\alpha}, i_{\alpha}-1\right\}$ for any $\alpha \in[k]$.

For example, $\Pi_{2}^{B}$ contains 6 partitions:

$$
\hat{0}^{B},\{\{ \pm 1, \pm 2\}\},\{ \pm\{1\},\{ \pm 2\}\},\{ \pm\{2\},\{ \pm 1\}\},\{ \pm\{1,2\}\},\{ \pm\{1,-2\}\} .
$$

Let $\pi=\{ \pm\{1\},\{ \pm 2\}\}$. We have $i_{0}=1, k=1$, and $i_{1}=1$. For $l=1$, by (2.1),

$$
N^{B}(\pi ; 1)=\sum_{i=0}^{1}\left[x^{i}\right](1+x)^{2}=3 .
$$

The three $B_{2}$-partitions which contain exactly 1 block pair and intersect $\pi$ minimally are $\{ \pm\{2\},\{ \pm 1\}\},\{ \pm\{1,2\}\}$, and $\{ \pm\{1,-2\}\}$. Recall that Pittel [7] characterized the intersecting structure of two partitions in terms of 01-matrices. He used the coefficient

$$
\left[\mathbf{x}^{\mathbf{i}} \mathbf{y}^{\mathbf{j}}\right] \prod_{\alpha \in[k], \beta \in[l]}\left(1+x_{\alpha} y_{\beta}\right)
$$

to represent the number of ways to assign 0 or 1 to all $k l$ pairwise intersections of blocks of two minimally intersecting ordinary partitions. We will use a similar idea to deal with the intersecting structure of $B_{n}$-partitions.

Proof of Theorem 2.1. Let $Z_{1}$ be the zero-block of $\pi$, and $\pm B_{1}, \pm B_{2}, \ldots, \pm B_{k}$ the block pairs of $\pi$. Let $Z_{2}$ be the zero-block of $\pi^{\prime}$, and $\pm B_{1}^{\prime}, \pm B_{2}^{\prime}, \ldots, \pm B_{l}^{\prime}$ the block pairs of $\pi^{\prime}$.

To ensure that $\pi$ and $\pi^{\prime}$ are minimally intersecting, it is necessary to characterize the intersecting relations for all pairs $\left(B, B^{\prime}\right)$ where $B$ is a block of $\pi$ and $B^{\prime}$ is a block of $\pi^{\prime}$. Since $\pi$ and $\pi^{\prime}$ intersect minimally, we observe that each $B \cap B^{\prime}$ contains at most one element, where both $B$ and $B^{\prime}$ may be the zero-block. So we have four cases.

- $B=Z_{1}$ and $B^{\prime}=Z_{2}$. We have $Z_{1} \cap Z_{2}=\emptyset$ since the cardinality of $Z_{1} \cap Z_{2}$ is even. 
- $B \neq Z_{1}$ and $B^{\prime}=Z_{2}$. We introduce the variable $z_{2}$ to represent the zero-block $Z_{2}$, and the variable $x_{\alpha}$ to represent the block $B_{\alpha}$. The intersection $B_{\alpha} \cap Z_{2}$ can be represented by $x_{\alpha} z_{2}$ if it is of cardinality 1 . In this case, the intersection $\left(-B_{\alpha}\right) \cap Z_{2}$ can be ignored since

$$
\left(-B_{\alpha}\right) \cap Z_{2}=-\left(B_{\alpha} \cap Z_{2}\right) .
$$

- $B=Z_{1}$ and $B^{\prime} \neq Z_{2}$. We introduce the variable $z_{1}$ to represent the zero-block $Z_{1}$, and the variable $w_{\beta}$ to represent the block $B_{\beta}^{\prime}$. Then $Z_{1} \cap B_{\beta}^{\prime}$ can be represented by $z_{1} w_{\beta}$ if it is of cardinality 1 . In this case, the intersection $Z_{1} \cap\left(-B_{\beta}^{\prime}\right)$ can be disregarded since

$$
Z_{1} \cap\left(-B_{\beta}^{\prime}\right)=-\left(Z_{1} \cap B_{\beta}^{\prime}\right)
$$

- $B \neq Z_{1}$ and $B^{\prime} \neq Z_{2}$. In this case, we introduce the variable $y_{\beta}$ (resp. $\bar{y}_{\beta}$ ) to represent the block $B_{\beta}^{\prime}$ (resp. $\quad-B_{\beta}^{\prime}$ ). Then $B_{\alpha} \cap B_{\beta}^{\prime}$ (resp. $B_{\alpha} \cap\left(-B_{\beta}^{\prime}\right)$ ) can be represented by $x_{\alpha} y_{\beta}$ (resp. $x_{\alpha} \bar{y}_{\beta}$ ) if it is of cardinality 1 . Note that it is not necessary to consider the intersection involving the block $-B_{\alpha}$ since

$$
\left(-B_{\alpha}\right) \cap\left( \pm B_{\beta}^{\prime}\right)=-\left(B_{\alpha} \cap\left(\mp B_{\beta}^{\prime}\right)\right)
$$

Combining the above four cases, we can represent the meet $\pi \wedge \pi^{\prime}$ by

$$
F(k ; l) \prod_{\alpha \in[k]}\left(1+x_{\alpha} z_{2}\right) \prod_{\beta \in[l]}\left(1+z_{1} w_{\beta}\right)
$$

where

$$
F(k ; l)=\prod_{\alpha \in[k], \beta \in[l]}\left(1+x_{\alpha} y_{\beta}\right)\left(1+x_{\alpha} \bar{y}_{\beta}\right) .
$$

Notice that the expression (2.3) is analogous to

$$
\prod_{\alpha \in[k], \beta \in[l]}\left(1+x_{\alpha} y_{\beta}\right)
$$

in (2.2). Now we are going to introduce an operator for (2.3) which corresponds to $\left[\mathbf{x}^{\mathbf{i}} \mathbf{y}^{\mathbf{j}}\right]$ in (2.2). In this way, we can express the number of ways to assign cardinalities 0 or 1 to all pairwise intersections of blocks of two minimally intersecting $B_{n}$-partitions.

Let $j_{0}$ be a nonnegative integer and $\mathbf{j}=\left(j_{1}, j_{2}, \ldots, j_{l}\right)$ a composition of $n-j_{0}$. Denote by $N^{B}\left(\pi ; j_{0}, \mathbf{j}\right)$ the number of $B_{n}$-partitions $\pi^{\prime}$ of type $\left(j_{0} ; \mathbf{j}\right)$ such that $\pi^{\prime}$ minimally meets $\pi$. In the above notation, we have

$$
N^{B}\left(\pi ; j_{0}, \mathbf{j}\right)=c \cdot \sum_{\mathbf{a}+\mathbf{b}+\mathbf{c}=\mathbf{j}}\left[\mathbf{x}^{\mathbf{i}} z_{1}^{i_{0}} z_{2}^{j_{0}} \mathbf{w}^{\mathbf{a}} \mathbf{y}^{\mathbf{b}} \overline{\mathbf{y}}^{\mathbf{c}}\right] F(k ; l) \prod_{\alpha \in[k]}\left(1+x_{\alpha} z_{2}\right) \prod_{\beta \in[l]}\left(1+z_{1} w_{\beta}\right),
$$

where

$$
c=\mathbf{i} ! \cdot \frac{\left(2 i_{0}\right) ! !}{(2 l) ! !}
$$


and

$$
\begin{array}{lll}
\mathbf{x}=\left(x_{1}, x_{2}, \ldots, x_{k}\right), & \mathbf{i}=\left(i_{1}, i_{2}, \ldots, i_{k}\right), & \mathbf{x}^{\mathbf{i}}=\prod_{\alpha \in[k]} x_{\alpha}^{i_{\alpha}} ; \\
\mathbf{w}=\left(w_{1}, w_{2}, \ldots, w_{l}\right), & \mathbf{a}=\left(a_{1}, a_{2}, \ldots, a_{l}\right), & \mathbf{w}^{\mathbf{a}}=\prod_{\beta \in[l]} w_{\beta}^{a_{\beta}} ; \\
\mathbf{y}=\left(y_{1}, y_{2}, \ldots, y_{l}\right), & \mathbf{b}=\left(b_{1}, b_{2}, \ldots, b_{l}\right), & \mathbf{y}^{\mathbf{b}}=\prod_{\beta \in[l]} y_{\beta}^{b_{\beta}} ; \\
\overline{\mathbf{y}}=\left(\bar{y}_{1}, \bar{y}_{2}, \ldots, \bar{y}_{l}\right), & \mathbf{c}=\left(c_{1}, c_{2}, \ldots, c_{l}\right), & \overline{\mathbf{y}}^{\mathbf{c}}=\prod_{\beta \in[l]} \bar{y}_{\beta}^{c_{\beta}} .
\end{array}
$$

Here we give a combinatorial explanation for the coefficient $c$ in (2.6). In fact, for the partition $\pi^{\prime}$, by permuting the $l$ block pairs or interchanging the two blocks in a common block pair, we still have the same partition. This explains the denominator ( $2 l) !$ !. On the other hand, for any block $B_{\alpha}$, every block of $\pi^{\prime}$ contains at most one element of $B_{\alpha}$. Considering the assignment of an element to the intersection $B_{\alpha} \cap B^{\prime}$, where $B^{\prime}$ is a block of $\pi^{\prime}$, we are led to the factor i!. Similarly, the factor $\left(2 i_{0}\right)$ !! is associated with the assignment of elements in $Z_{1}$ to the blocks of $\pi^{\prime}$.

Denote by $\left(\begin{array}{c}S \\ m\end{array}\right)$ the collection of all $m$-subsets of $S$. Since

$$
\begin{aligned}
& {\left[z_{2}^{j_{0}}\right] \prod_{\alpha \in[k]}\left(1+x_{\alpha} z_{2}\right)=\sum_{X \in\left(\begin{array}{c}
{[k]} \\
j_{0}
\end{array}\right)} \prod_{\alpha \in X} x_{\alpha},} \\
& {\left[z_{1}^{i_{0}}\right] \prod_{\beta \in[l]}\left(1+z_{1} w_{\beta}\right)=\sum_{Y \in\left(\begin{array}{c}
{[l]} \\
i_{0}
\end{array}\right)} \prod_{\beta \in Y} w_{\beta},}
\end{aligned}
$$

substituting (2.7) and (2.8) into (2.5), we obtain that

$$
\begin{aligned}
N^{B}\left(\pi ; j_{0}, \mathbf{j}\right) & =c \cdot \sum_{\mathbf{a}+\mathbf{b}+\mathbf{c}=\mathbf{j}}\left[\mathbf{x}^{\mathbf{i}} \mathbf{w}^{\mathbf{a}} \mathbf{y}^{\mathbf{b}} \overline{\mathbf{y}}^{\mathbf{c}}\right]\left(\sum_{Y \in\left(\begin{array}{l}
{[l]} \\
i_{0}
\end{array}\right)} \prod_{\beta \in Y} w_{\beta}\right)\left(\sum_{X \in\left(\begin{array}{l}
{[k]} \\
j_{0}
\end{array}\right)} \prod_{\alpha \in X} x_{\alpha}\right) F(k ; l) \\
& =c \cdot \sum_{X, Y, \mathbf{b}}\left[\mathbf{y}^{\mathbf{b}} \prod_{\alpha \in[k]} x_{\alpha}^{i_{\alpha}-\chi(\alpha \in X)} \prod_{\beta \in[l]} \bar{y}_{\beta}^{j_{\beta}-b_{\beta}-\chi(\beta \in Y)}\right] F(k ; l),
\end{aligned}
$$

where $\chi$ is defined by $\chi(P)=1$ if $P$ is true, and $\chi(P)=0$ otherwise. Therefore the number of $B_{n}$-partitions $\pi^{\prime}$ containing exactly $l$ block pairs that intersect $\pi$ minimally equals

$$
N^{B}(\pi ; l)=\sum_{\substack{j_{0}+j_{1}+\cdots+j_{l}=n \\ j_{0} \geqslant 0, j_{1}, \ldots, j_{l} \geqslant 1}} N^{B}\left(\pi ; j_{0}, \mathbf{j}\right)=c \cdot \sum_{j_{0}, X}\left[\prod_{\alpha} x_{\alpha}^{i_{\alpha}-\chi(\alpha \in X)}\right] \sum_{\substack{j_{0}+j_{1}+\cdots+j_{l}=n \\ j_{1}, \ldots, j_{l} \geqslant 1}} f(\mathbf{j}),
$$

where

$$
f(\mathbf{j})=\sum_{Y, \mathbf{b}}\left[\mathbf{y}^{\mathbf{b}} \prod_{\beta} \bar{y}_{\beta}^{j_{\beta}-b_{\beta}-\chi(\beta \in Y)}\right] F(k ; l)
$$


In view of the expression (2.4), the total degree of $x_{\alpha}$ in $F(k ; l)$ agrees with the sum of the degrees of $y_{\beta}$ and $\bar{y}_{\beta}$. Concerning (2.9), we find

$$
\sum_{\alpha \in[k]} i_{\alpha}-\chi(\alpha \in X)=\sum_{\beta \in[l]} b_{\beta}+\left(j_{\beta}-b_{\beta}-\chi(\beta \in Y)\right),
$$

that is,

$$
j_{0}+j_{1}+\cdots+j_{l}=i_{0}+i_{1}+\cdots+i_{k}=n .
$$

So we may drop this condition in the inner summation of (2.9). In order to reduce the factor $\sum_{j_{1}, \ldots, j_{l} \geqslant 1} f(\mathbf{j})$, we introduce

$$
S(A)=\sum_{\substack{j_{1}, \ldots, j_{l} \geqslant 0 \\ j_{\beta}=0 \text { if } \beta \notin A}} f(\mathbf{j})=\sum_{Y} \sum_{\substack{b_{\gamma}, j_{\gamma} \geqslant 0 \\ \gamma \in A}}\left[\prod_{\gamma \in A} y_{\gamma}^{b_{\gamma}} \bar{y}_{\gamma}^{j_{\gamma}-b_{\gamma}-\chi(\gamma \in Y)}\right] F(k ; A)
$$

for any $A \subseteq[l]$, where

$$
F(k ; A)=\prod_{\alpha \in[k], \gamma \in A}\left(1+x_{\alpha} y_{\gamma}\right)\left(1+x_{\alpha} \bar{y}_{\gamma}\right)
$$

Since $j_{\gamma}$ and $b_{\gamma}$ run over all nonnegative integers, the exponent $j_{\gamma}-b_{\gamma}-\chi(\gamma \in Y)$ can be considered as a summation index. It follows that

$$
S(A)=\sum_{Y \in\left(\begin{array}{c}
A \\
i_{0}
\end{array}\right)} \sum_{b_{\gamma}, c_{\gamma} \geqslant 0, \gamma \in A}\left[\prod_{\gamma \in A} y_{\gamma}^{b_{\gamma}} \bar{y}_{\gamma}^{c_{\gamma}}\right] F(k ; A)=\left(\begin{array}{c}
|A| \\
i_{0}
\end{array}\right) \prod_{\alpha \in[k]}\left(1+x_{\alpha}\right)^{2|A|} .
$$

By the principle of inclusion-exclusion, we have

$$
\begin{aligned}
\sum_{j_{1}, \ldots, j_{l} \geqslant 1} f(\mathbf{j}) & =\sum_{A \subseteq[l]}(-1)^{l-|A|} S(A)=\sum_{m}\left(\begin{array}{c}
l \\
m
\end{array}\right)(-1)^{l-m}\left(\begin{array}{c}
m \\
i_{0}
\end{array}\right) \prod_{\alpha \in[k]}\left(1+x_{\alpha}\right)^{2 m} \\
& =\left(\begin{array}{c}
l \\
i_{0}
\end{array}\right) \prod_{\alpha \in[k]}\left(1+x_{\alpha}\right)^{2 i_{0}}\left(\prod_{\alpha \in[k]}\left(1+x_{\alpha}\right)^{2}-1\right)^{l-i_{0}}
\end{aligned}
$$

Now, employing $(2.9)$ we find that $N^{B}(\pi ; l)$ equals

$$
\frac{\mathbf{i} !}{\left(2 l-2 i_{0}\right) ! !} \sum_{X \subseteq[k]}\left[\prod_{\alpha \in[k]} x_{\alpha}^{i_{\alpha}-\chi(\alpha \in X)}\right] \prod_{\alpha \in[k]}\left(1+x_{\alpha}\right)^{2 i_{0}}\left(\prod_{\alpha \in[k]}\left(1+x_{\alpha}\right)^{2}-1\right)^{l-i_{0}}
$$

which can be rewritten in the form of (2.1). This completes the proof. 
Summing (2.1) over $l \geqslant i_{0}$, we obtain the following formula.

Corollary 2.2 The number $N^{B}(\pi)$ of $B_{n}$-partitions that minimally intersect $\pi$ is

$$
N^{B}(\pi)=\frac{\mathbf{i} !}{\sqrt{e}} \sum_{\mathbf{i}^{\prime}}\left[\mathbf{x}^{\mathbf{i}^{\prime}}\right] F(\mathbf{x})
$$

where

$$
F(\mathbf{x})=\left(\prod_{\alpha \in[k]}\left(1+x_{\alpha}\right)^{2 i_{0}}\right) \exp \left(\frac{1}{2} \prod_{\alpha \in[k]}\left(1+x_{\alpha}\right)^{2}\right)
$$

Setting $\pi=\hat{0}^{B}$ in (2.11), we get $i_{0}=0$ and

$$
N^{B}\left(\hat{0}^{B}\right)=\frac{1}{\sqrt{e}} \sum_{i_{\alpha}^{\prime} \in\{0,1\}}\left[x_{1}^{i_{1}^{\prime}} \cdots x_{n}^{i_{n}^{\prime}}\right] \sum_{j \geqslant 0} \frac{1}{(2 j) ! !} \prod_{\alpha=1}^{n}\left(1+x_{\alpha}\right)^{2 j} .
$$

This immediately reduces to Benoumhani's formula for the Dowling number

$$
\left|\Pi_{n}^{B}\right|=\frac{1}{\sqrt{e}} \sum_{k \geqslant 0} \frac{(2 k+1)^{n}}{(2 k) ! !}
$$

in analogy to Dobiński's formula (1.2). In fact, the number $N^{B}(\pi)$ can also be written as an infinite sum.

\section{Corollary 2.3}

$$
N^{B}(\pi)=\frac{1}{\sqrt{e}} \sum_{j \geqslant 0} \frac{\left(2 i_{0}+2 j+1\right) !^{k}}{(2 j) ! !} \prod_{\alpha \in[k]} \frac{1}{\left(2 i_{0}+2 j+1-i_{\alpha}\right) !} .
$$

Proof. From (2.12) it follows that

$$
F(x)=\sum_{j \geqslant 0} \frac{1}{(2 j) ! !} \prod_{\alpha \in[k]}\left(1+x_{\alpha}\right)^{2\left(i_{0}+j\right)} .
$$

Hence

$$
\begin{aligned}
N^{B}(\pi) & =\frac{\mathbf{i} !}{\sqrt{e}} \sum_{j \geqslant 0} \frac{1}{(2 j) ! !} \prod_{\alpha \in[k]}\left(\left(\begin{array}{c}
2\left(i_{0}+j\right) \\
i_{\alpha}
\end{array}\right)+\left(\begin{array}{c}
2\left(i_{0}+j\right) \\
i_{\alpha}-1
\end{array}\right)\right) \\
& =\frac{\mathbf{i} !}{\sqrt{e}} \sum_{j \geqslant 0} \frac{1}{(2 j) ! !} \prod_{\alpha \in[k]}\left(\begin{array}{c}
2\left(i_{0}+j\right)+1 \\
i_{\alpha}
\end{array}\right),
\end{aligned}
$$

which gives (2.14). This completes the proof. 
Corollary 2.4 Let $N_{n, 2}^{B}\left(i_{0} ; k\right)$ denote the number of ordered pairs $\left(\pi, \pi^{\prime}\right)$ of minimally intersecting $B_{n}$-partitions such that $\pi$ consists of exactly $k$ block pairs and a zero-block of half-size $i_{0}$ (allowing $i_{0}=0$ ). Then

$$
N_{n, 2}^{B}\left(i_{0} ; k\right)=\frac{(2 n) ! !}{\left(2 i_{0}\right) ! !(2 k) ! ! \sqrt{e}}\left[x^{n-i_{0}}\right] \sum_{j \geqslant 0} \frac{1}{(2 j) ! !}\left((1+x)^{2 i_{0}+2 j+1}-1\right)^{k} .
$$

Proof. By a simple combinatorial argument, we see that the number of $B_{n}$-partitions of type $\left(i_{0} ; i_{1}, \ldots, i_{k}\right)$ equals

$$
c=\left(\begin{array}{c}
n \\
i_{0}, i_{1}, \ldots, i_{k}
\end{array}\right) \frac{2^{n-i_{0}-k}}{k !}=\frac{(2 n) ! !}{\left(2 i_{0}\right) ! !(2 k) ! !} \cdot \frac{1}{\mathbf{i} !} .
$$

Thus by (2.11), we have

$$
N_{n, 2}^{B}(k)=\sum_{\substack{i_{0}+i_{1}+\cdots+i_{k}=n \\ i_{1}, \ldots, i_{k} \geqslant 1}} c \cdot N^{B}(\pi)=\frac{(2 n) ! !}{\left(2 i_{0}\right) ! !(2 k) ! ! \sqrt{e}} \sum_{\substack{i_{0}+i_{1}+\cdots+i_{k}=n \\ i_{1}, \ldots, i_{k} \geqslant 1}} \sum_{\mathbf{i}^{\prime}}\left[\mathbf{x}^{\mathbf{i}^{\prime}}\right] F(\mathbf{x}) .
$$

For any $A \subseteq[k]$, consider

$$
S(A)=\sum_{\substack{i_{0}+i_{1}+\cdots+i_{k}=n \\ i_{1}, \ldots, i_{k} \geqslant 0 \\ i_{\alpha}=0 \text { if } \alpha \notin A}} \sum_{\mathbf{i}^{\prime}}\left[\mathbf{x}^{\mathbf{i}^{\prime}}\right] F(\mathbf{x})=\sum_{\substack{i_{0}+\sum_{\alpha \in A} i_{\alpha}=n \\ i_{\alpha} \geqslant 0, \alpha \in A}} \sum_{\left.\mathbf{i}^{\prime}\right|_{A}}\left[\left.\mathbf{x}^{\mathbf{i}^{\prime}}\right|_{A}\right] F\left(\left.\mathbf{x}\right|_{A}\right)
$$

where $\left.\mathbf{x}\right|_{A}\left(\right.$ resp. $\left.\left.\mathbf{i}^{\prime}\right|_{A}\right)$ denotes the vector obtained by removing all $x_{\alpha}$ (resp. $\left.i_{\alpha}^{\prime}\right)$ such that $\alpha \notin A$ from the vector $\mathbf{x}$ (resp. $\mathbf{i}^{\prime}$ ). Let $t$ be the number of $\alpha$ 's such that $i_{\alpha}^{\prime}=i_{\alpha}-1$ in the inner summation. Noting that

$$
F\left(\left.\mathbf{x}\right|_{A}\right)=\left(\prod_{\alpha \in A}\left(1+x_{\alpha}\right)^{2 i_{0}}\right) \exp \left(\frac{1}{2} \prod_{\alpha \in A}\left(1+x_{\alpha}\right)^{2}\right)
$$

$S(A)$ can be written as

$$
\begin{aligned}
S(A) & =\left(\sum_{t}\left(\begin{array}{c}
|A| \\
t
\end{array}\right)\left[x^{n-i_{0}-t}\right]\right)(1+x)^{2 i_{0}|A|} \exp \left(\frac{1}{2}(1+x)^{2|A|}\right) \\
& =\left[x^{n-i_{0}}\right](1+x)^{\left(2 i_{0}+1\right)|A|} \exp \left(\frac{1}{2}(1+x)^{2|A|}\right) .
\end{aligned}
$$

In view of the principle of inclusion-exclusion, we deduce from (2.16) that

$$
N_{n, 2}^{B}(k)=\frac{(2 n) ! !}{\left(2 i_{0}\right) ! !(2 k) ! ! \sqrt{e}} \sum_{A \subseteq[k]}(-1)^{k-|A|} S(A),
$$

which gives (2.15). This completes the proof. 
Summing over $0 \leqslant k \leqslant n-i_{0}$ and $0 \leqslant i_{0} \leqslant n$, we obtain the number of ordered pairs of minimally intersecting $B_{n}$-partitions.

Corollary 2.5 The number $N_{n, 2}^{B}$ of ordered pairs $\left(\pi, \pi^{\prime}\right)$ of minimally intersecting $B_{n^{-}}$ partitions is given by

$$
N_{n, 2}^{B}=\frac{2^{n}}{e} \sum_{k, l \geqslant 0} \frac{(2 k l+k+l)_{n}}{(2 k) ! !(2 l) ! !} .
$$

For example, $N_{1,2}^{B}=3, N_{2,2}^{B}=23, N_{3,2}^{B}=329$. For general $r$, we have Theorem 1.1. We now proceed to give a proof as a direct generalization of the proof of Corollary 2.5. Proof of Theorem 1.1. For any $s \in[r]$, let $i_{s}$ be an nonnegative integer and $\mathbf{j}_{s}=$ $\left(j_{s, 1}, j_{s, 2}, \ldots, j_{s, k_{s}}\right)$ be a composition of $n$. Let $\pi_{s}$ be a $B_{n}$-partition of type $\left(i_{s} ; \mathbf{j}_{s}\right)$, with the zero-block $Z_{s}$ and block pairs

$$
\pm B_{s, 1}, \pm B_{s, 2}, \ldots, \pm B_{s, k_{s}}
$$

Suppose that $\pi_{1}, \pi_{2}, \ldots, \pi_{r}$ are minimally intersecting. Let $B_{s}$ be a block of $\pi_{s}(1 \leqslant s \leqslant r)$. It may be either the zero-block $Z_{s}$ or any one of the $2 k_{s}$ blocks in (2.17). We shall consider each intersection

$$
B_{1} \cap B_{2} \cap \cdots \cap B_{r} .
$$

Since $\pi_{1}, \pi_{2}, \ldots, \pi_{r}$ are minimally intersecting, each intersection (2.18) contains at most one element. We consider the number $t \in\{0,1, \ldots, r+1\}$ such that

$$
B_{1}=Z_{1}, B_{2}=Z_{2}, \ldots, B_{t-1}=Z_{t-1}, B_{t} \neq Z_{t} .
$$

In particular, the case $t=0$ (resp. $t=r+1$ ) implies that all $B_{s}$ 's are non-zero-blocks (resp. zero-blocks). Note that

$$
\bigcap_{s \in[t-1]} Z_{s} \cap\left(-B_{t}\right)=-\left(\bigcap_{s \in[t-1]} Z_{s} \cap B_{t}\right) .
$$

So the intersection in the form of (2.18) can be excluded when $B_{t}=-B_{t, i}$ for some $i \in\left[k_{t}\right]$.

We now assume that $B_{t}=B_{t, i}$ for some $i$. We use the variable $z_{s}$ to represent $Z_{s}$ for all $s \in[r]$, and use $x_{t, i}$ to represent the block $B_{t, i}$. For $p \geqslant t+1$, we use the variable $y_{p, i}$ (resp. $\bar{y}_{p, i}$ ) to represent the block $B_{p, i}$ (resp. $-B_{p, i}$ ), where $i \in\left[k_{p}\right]$. So we can represent the intersection property by a factor

$$
f_{t}=1+z_{1} \cdots z_{t-1} x_{t, \alpha_{t}} Y_{t+1} \cdots Y_{r}
$$

where $\alpha_{t} \in\left[k_{t}\right]$ and

$$
Y_{p} \in\left\{z_{p}, y_{p, 1}, \bar{y}_{p, 1}, \ldots, y_{p, k_{p}}, \bar{y}_{p, k_{p}}\right\}
$$


for any $p \geqslant t+1$. Let

$$
\begin{array}{lll}
\mathbf{x}_{s}=\left(x_{s, 1}, \ldots, x_{s, k_{s}}\right), & \mathbf{a}_{s}=\left(a_{s, 1}, \ldots, a_{s, k_{s}}\right), & \mathbf{x}_{s}^{\mathbf{a}_{s}}=\prod_{i \in\left[k_{s}\right]} x_{s, i}^{a_{s, i}} ; \\
\mathbf{y}_{s}=\left(y_{s, 1}, \ldots, y_{s, k_{s}}\right), & \mathbf{b}_{s}=\left(b_{s, 1}, \ldots, b_{s, k_{s}}\right), & \mathbf{y}_{s}^{\mathbf{b}_{s}}=\prod_{i \in\left[k_{s}\right]} y_{s, i}^{b_{s, i}} ; \\
\overline{\mathbf{y}}_{s}=\left(\bar{y}_{s, 1}, \ldots, \bar{y}_{s, k_{s}}\right), & \mathbf{c}_{s}=\left(c_{s, 1}, \ldots, c_{s, k_{s}}\right), & \overline{\mathbf{y}}_{s}^{\mathbf{c}_{s}}=\prod_{i \in\left[k_{s}\right]} \bar{y}_{s, i}^{c_{s, i}} .
\end{array}
$$

Denote by $N^{B}\left(\pi_{1} ; i_{2}, \mathbf{j}_{2} ; \ldots ; i_{r}, \mathbf{j}_{r}\right)$ the number of $(r-1)$-tuples $\left(\pi_{2}, \ldots, \pi_{r}\right)$ of $B_{n}$-partitions such that $\pi_{s}(2 \leqslant s \leqslant r)$ is of type $\left(i_{s}, \mathbf{j}_{s}\right)$ and $\pi_{1}, \pi_{2}, \ldots, \pi_{r}$ intersect minimally. In the notation of $f_{t}$ in $(2.19)$, we get

$$
N^{B}\left(\pi_{1} ; i_{2}, \mathbf{j}_{2} ; \ldots ; i_{r}, \mathbf{j}_{r}\right)=c\left[\mathbf{x}_{1}^{\mathbf{j}_{1}} z_{1}^{i_{1}}\right] \sum_{\substack{\mathbf{a}_{s}+\mathbf{b}_{s}+\mathbf{c}_{s}=\mathbf{j}_{s} \\ 2 \leqslant s \leqslant r}}\left[\mathbf{x}_{s}^{\mathbf{a}_{s}} \mathbf{y}_{s}^{\mathbf{b}_{s}} \overline{\mathbf{y}}_{s}^{\mathbf{c}_{s}} z_{s}^{i_{s}}\right] F_{r},
$$

where

$$
\begin{aligned}
& c=\mathbf{j}_{1} ! \cdot\left(2 i_{1}\right) ! ! \prod_{2 \leqslant s \leqslant r}\left(2 k_{s}\right) ! !^{-1}, \\
& F_{r}=\prod_{t \in[r]} \prod_{\alpha_{t} \in\left[k_{t}\right]} \prod_{\substack{Y_{p} \in\left\{\left\{z_{p}, y_{p, 1}, \bar{p}_{p, 1}, \ldots, y_{\left.p, k_{p}, \bar{y}_{p, k_{p}}\right\}}\right\} \\
t+1 \leqslant p \leqslant r\right.}} f_{t} .
\end{aligned}
$$

The value of the coefficient $c$ in (2.20) can be explained similar to the one in (2.6). We omit the explanation here.

Now, let $N^{B}\left(\pi_{1}, k_{2}, \ldots, k_{r}\right)$ be the number of $(r-1)$-tuples $\left(\pi_{2}, \ldots, \pi_{r}\right)$ of $B_{n}$-partitions such that $\pi_{s}$ contains exactly $k_{s}$ block pairs and $\pi_{1}, \pi_{2}, \ldots, \pi_{r}$ intersect minimally. Then

$$
N^{B}\left(\pi_{1}, k_{2}, \ldots, k_{r}\right)=\sum_{\substack{i_{s} \geqslant 0, j_{s, 1}, \ldots, j_{s, k} \geqslant 1 \\ j_{s, 1}+\cdots+j_{s, k_{s}+i_{s}=n}}} N^{B}\left(\pi_{1} ; i_{2}, \mathbf{j}_{2} ; \ldots ; i_{r}, \mathbf{j}_{r}\right) .
$$

We claim that the conditions $j_{s, 1}+\cdots+j_{s, k_{s}}+i_{s}=n$ can be dropped in the above summation. In fact, for any $i \in\{1,2, \ldots, r\}$, the sum of the degrees of $\mathbf{x}_{i}, \mathbf{y}_{i}, \overline{\mathbf{y}}_{i}$, and $z_{i}$ is 0 or 1 in the factor $f_{t}$. More importantly, this sum is independent of $i$. In particular, the sum for $i=1$ equals the sum for any $2 \leqslant s \leqslant r$, that is,

$$
j_{s, 1}+\cdots+j_{s, k_{s}}+i_{s}=j_{1,1}+\cdots+j_{1, k_{1}}+i_{1}=n .
$$

Hence we can ignore the conditions (2.22) in (2.21). This implies that

$$
N^{B}\left(\pi_{1}, k_{2}, \ldots, k_{r}\right)=c\left[\mathbf{x}_{1}^{\mathbf{j}_{1}} z_{1}^{i_{1}}\right] \sum_{\substack{i_{s} \geqslant 0, \mathbf{a}_{s}+\mathbf{b}_{s}+\mathbf{c}_{s} \geqslant 1 \\ 2 \leqslant s \leqslant r}}\left[\mathbf{x}_{s}^{\mathbf{a}_{s}} \mathbf{y}_{s}^{\mathbf{b}_{s}} \overline{\mathbf{y}}_{s}^{\mathbf{c}_{s}} z_{s}^{i_{s}}\right] F_{r},
$$


where $\mathbf{a}_{s}+\mathbf{b}_{s}+\mathbf{c}_{s} \geqslant 1$ indicates that $a_{s, h_{s}}+b_{s, h_{s}}+c_{s, h_{s}} \geqslant 1$ for any $1 \leqslant h_{s} \leqslant k_{s}$. We will compute $\sum\left[\mathbf{x}_{s}^{\mathbf{a}_{s}} \mathbf{y}_{s}^{\mathbf{b}_{s}} \overline{\mathbf{y}}_{s}^{\mathbf{c}_{s}} z_{s}^{i_{s}}\right] F_{r}$ for $s=2,3, \ldots, r$ by the following procedure. First, for $s=2$, we have

$$
\sum_{i_{2} \geqslant 0, \mathbf{a}_{2}+\mathbf{b}_{2}+\mathbf{c}_{2} \geqslant \mathbf{1}}\left[\mathbf{x}_{2}^{\mathbf{a}_{2}} \mathbf{y}_{2}^{\mathbf{b}_{2}} \overline{\mathbf{y}}_{2}^{\mathbf{c}_{2}} z_{2}^{i_{2}}\right] F_{r}=\sum_{l_{2}}\left(\begin{array}{c}
k_{2} \\
l_{2}
\end{array}\right)(-1)^{k_{2}-l_{2}} F_{r, 2}
$$

where $F_{r, 2}$ equals

$$
\prod_{\alpha_{1}, Y_{p}}\left(1+x_{1, \alpha_{1}} Y_{3} \cdots Y_{r}\right)^{2 l_{2}+1} \prod_{Y_{p}}\left(1+z_{1} Y_{3} \cdots Y_{r}\right)^{l_{2}} \prod_{t \geqslant 3, \alpha_{t}, Y_{p}}\left(1+z_{1} z_{3} \cdots z_{t-1} x_{t, \alpha_{t}} Y_{t+1} \cdots Y_{r}\right)
$$

So $N^{B}\left(\pi_{1}, k_{2}, \ldots, k_{r}\right)$ equals

$$
c\left[\mathbf{x}_{1}^{\mathbf{j}_{1}} z_{1}^{i_{1}}\right] \sum_{l_{2}}\left(\begin{array}{c}
k_{2} \\
l_{2}
\end{array}\right)(-1)^{k_{2}-l_{2}} \sum_{\substack{i_{s} \geqslant 0, \mathbf{a}_{s}+\mathbf{b}_{s}+\mathbf{c}_{s} \geqslant 1 \\
3 \leqslant s \leqslant r}}\left[\mathbf{x}_{s}^{\mathbf{a}_{s}} \mathbf{y}_{s}^{\mathbf{b}_{s}} \overline{\mathbf{y}}_{s}^{\mathbf{c}_{s}} z_{s}^{i_{s}}\right] F_{r, 2} .
$$

To compute the inner summation, let

$$
g_{s}=\frac{1}{2}\left(\prod_{2 \leqslant i \leqslant s}\left(2 l_{i}+1\right)-1\right)
$$

For any $s \geqslant 2$, it is clear that

$$
\left(2 l_{s+1}+1\right) g_{s}+l_{s+1}=g_{s+1} .
$$

Starting with (2.23), we can continue the above procedure to deduce that for any $2 \leqslant$ $h \leqslant r-1, N^{B}\left(\pi_{1}, k_{2}, \ldots, k_{r}\right)$ equals

$$
c\left[\mathbf{x}_{1}^{\mathbf{j}_{1}} z_{1}^{i_{1}}\right] \sum_{l_{2}, \ldots, l_{h}} \prod_{2 \leqslant i \leqslant h}\left(\begin{array}{c}
k_{i} \\
l_{i}
\end{array}\right)(-1)^{k_{i}-l_{i}} \sum_{\substack{i_{s} \geqslant 0, \mathbf{a}_{s}+\mathbf{b}_{s}+\mathbf{c}_{s} \geqslant 1 \\
h+1 \leqslant s \leqslant r}}\left[\mathbf{x}_{s}^{\mathbf{a}_{s}} \mathbf{y}_{s}^{\mathbf{b}_{s}} \overline{\mathbf{y}}_{s}^{\mathbf{c}_{s}} z_{s}^{i_{s}}\right] F_{r, h},
$$

where

$$
\begin{aligned}
F_{r, h}= & \prod_{\alpha_{1}, Y_{p}}\left(1+x_{1, \alpha_{1}} Y_{h+1} \cdots Y_{r}\right)^{\prod_{2 \leqslant i \leqslant h}\left(2 l_{i}+1\right)} \prod_{Y_{p}}\left(1+z_{1} Y_{h+1} \cdots Y_{r}\right)^{g_{h}} \\
& \cdot \prod_{t \geqslant h+1, \alpha_{t}, Y_{p}}\left(1+z_{1} z_{h+1} \cdots z_{t-1} x_{t, \alpha_{t}} Y_{t+1} \cdots Y_{r}\right) .
\end{aligned}
$$

In particular, for $h=r-1$, we have

$$
N^{B}\left(\pi_{1}, k_{2}, \ldots, k_{r}\right)=c\left[\mathbf{x}_{1}^{\mathbf{j}_{1}} z_{1}^{i_{1}}\right] \sum_{l_{2}, \ldots, l_{r-1}}\left(\prod_{2 \leqslant i \leqslant r-1}\left(\begin{array}{c}
k_{i} \\
l_{i}
\end{array}\right)(-1)^{k_{i}-l_{i}}\right) G
$$


where

$$
\begin{aligned}
G & =\sum_{\mathbf{a}_{r}+\mathbf{b}_{r}+\mathbf{c}_{r} \geqslant \mathbf{1}}\left[\mathbf{x}_{r}^{\mathbf{a}_{r}} \mathbf{y}_{r}^{\mathbf{b}_{r}} \overline{\mathbf{y}}_{r}^{\mathbf{c}_{r}}\right] \prod_{\alpha_{1}, Y_{p}}\left(1+x_{1, \alpha_{1}}\right)^{\prod_{2 \leqslant i \leqslant r-1}\left(2 l_{i}+1\right)} \prod_{Y_{p}}\left(1+z_{1}\right)^{g_{r-1}} \prod_{\alpha_{r}}\left(1+z_{1} x_{r, \alpha_{r}}\right) \\
& =\sum_{l_{r}}\left(\begin{array}{c}
k_{r} \\
l_{r}
\end{array}\right)(-1)^{k_{r}-l_{r}}\left(1+z_{1}\right)^{g_{r}} \prod_{\alpha_{1}}\left(1+x_{1, \alpha_{1}}\right)^{\prod_{2 \leqslant i \leqslant r}\left(2 l_{i}+1\right)} .
\end{aligned}
$$

Since the number of $B_{n}$-partitions of type $\mathbf{j}_{1}$ equals

$$
c^{\prime}=\left(\begin{array}{c}
n \\
i_{1}
\end{array}\right)\left(\begin{array}{c}
n-i_{1} \\
\mathbf{j}_{1}
\end{array}\right) \frac{2^{n-i_{1}-k_{1}}}{k_{1} !}=\frac{(2 n) ! !}{\left(2 i_{1}\right) ! !\left(2 k_{1}\right) ! ! \mathbf{j}_{1} !},
$$

by $(2.24)$, we obtain

$$
\begin{aligned}
N_{n, r}^{B} & =\sum_{\substack{j_{1,1}, \ldots, j_{1, k_{1}} \geqslant 1 \\
i_{1}+j_{1,1}+\cdots+j_{1, k_{1}}=n}} c^{\prime} \sum_{k_{2}, \ldots, k_{r}} N^{B}\left(\pi_{1}, k_{2}, \ldots, k_{r}\right) \\
& =(2 n) ! ! \sum_{\substack{k_{2}, \ldots, k_{r} \\
l_{2}, \ldots, l_{r}}}\left(\prod_{2 \leqslant s \leqslant r}\left(\begin{array}{c}
k_{s} \\
l_{s}
\end{array}\right) \frac{(-1)^{k_{s}-l_{s}}}{\left(2 k_{s}\right) ! !}\right) \sum_{i_{1}, k_{1}} \frac{1}{\left(2 k_{1}\right) ! !}\left[z_{1}^{i_{1}}\right]\left(1+z_{1}\right)^{g_{r}} H,
\end{aligned}
$$

where

$$
\begin{aligned}
H & =\sum_{\substack{i_{1}+j_{1}, 1+\cdots+j_{1, k_{1}}=n \\
j_{1,1}, j_{1,2}, \ldots, j_{1, k_{1}} \geqslant 1}}\left[\mathbf{x}_{1}^{\mathbf{j}_{1}}\right] \prod_{\alpha_{1}}\left(1+x_{1, \alpha_{1}}\right)^{\prod_{2 \leqslant i \leqslant r}\left(2 l_{i}+1\right)} \\
& =\sum_{l_{1}}\left(\begin{array}{c}
k_{1} \\
l_{1}
\end{array}\right)(-1)^{k_{1}-l_{1}}\left[x^{n-i_{1}}\right](1+x)^{l_{1} \prod_{2 \leqslant i \leqslant r}\left(2 l_{i}+1\right)} .
\end{aligned}
$$

Using the identity

$$
\sum_{k}\left(\begin{array}{l}
k \\
l
\end{array}\right) \frac{(-1)^{k-l}}{(2 k) ! !}=\frac{e^{-1 / 2}}{(2 l) ! !}
$$

we can simplify the summation over $k_{1}, k_{2}, \ldots, k_{r} \geqslant 0$ in (2.25) in the following way.

$$
\begin{aligned}
N_{n, r}^{B} & =(2 n) ! ! \sum_{\substack{k_{1}, k_{2}, \ldots, k_{r} \\
l_{1}, l_{2}, \ldots, l_{r}}}\left(\prod_{t \in[r]}\left(\begin{array}{c}
k_{t} \\
l_{t}
\end{array}\right) \frac{(-1)^{k_{t}-l_{t}}}{\left(2 k_{t}\right) ! !}\right) \sum_{i_{1}}\left[x^{n-i_{1}} z_{1}^{i_{1}}\right]\left(1+z_{1}\right)^{g_{r}}(1+x)^{l_{1} \prod_{2 \leqslant i \leqslant r}\left(2 l_{i}+1\right)} \\
& =\frac{(2 n) ! !}{e^{r / 2}} \sum_{l_{1}, l_{2}, \ldots, l_{r}} \frac{1}{\left(2 l_{1}\right) ! !\left(2 l_{2}\right) ! ! \cdots\left(2 l_{r}\right) ! !}\left[x^{n}\right](1+x)^{g_{r}+l_{1} \prod_{2 \leqslant i \leqslant r}\left(2 l_{i}+1\right)} .
\end{aligned}
$$

To further simplify the above summation, we observe that

$$
g_{r}+l_{1} \prod_{2 \leqslant i \leqslant r}\left(2 l_{i}+1\right)=\frac{1}{2}\left(\prod_{t \in[r]}\left(2 l_{t}+1\right)-1\right) .
$$

Substituting (2.28) into (2.27), we arrive at (1.7). This completes the proof.

For example, we have $N_{1, r}=2^{r}-1$ and $N_{2,3}^{B}=187$. 


\section{Minimally intersecting $B_{n}$-partitions without zero- block}

In this section, we consider $B_{n}$-partitions without zero-block and give analogous results for the minimally intersecting problems which was investigated in the last section. Clearly $B_{n}$-partitions without zero-block form a meet-semilattice under refinement. The minimal $B_{n}$-partition without zero-block is still $\hat{0}^{B}$. We will omit the redundant proofs.

Inspecting the proof of Theorem 2.1, we can restrict our attention to the $B_{n}$-partitions without zero-block by setting $i_{0}=0$ and $X=\emptyset$ in (2.10). Concretely speaking, let $\pi$ be a $B_{n}$-partition consisting of $k$ block pairs of sizes $i_{1}, i_{2}, \ldots, i_{k}$ listed in any order. For a given $l \geqslant 1$, the number $N^{D}(\pi ; l)$ of $B_{n}$-partitions $\pi^{\prime}$ consisting of $l$ block pairs, which intersect $\pi$ minimally, is equal to

$$
N^{D}(\pi ; l)=\frac{\mathbf{i} !}{(2 l) ! !}\left[\mathbf{x}^{\mathbf{i}}\right]\left(\prod_{\alpha \in[k]}\left(1+x_{\alpha}\right)^{2}-1\right)^{l} .
$$

The number of $B_{n}$-partitions without zero-block that intersect $\pi$ minimally is given by

$$
N^{D}(\pi)=\frac{\mathbf{i} !}{\sqrt{e}}\left[\mathbf{x}^{\mathbf{i}]} \exp \left(\frac{1}{2} \prod_{\alpha \in[k]}\left(1+x_{\alpha}\right)^{2}\right) .\right.
$$

For example, let $n=3, \pi=\{ \pm\{2\}, \pm\{1,-3\}\}$ and $l=2$. Then $(3.1)$ yields $N^{D}(\pi ; 2)=5$. In fact, the $B_{n}$-partitions consisting of 2 block pairs which intersect $\pi$ minimally are exactly the 5 partitions consisting of two block pairs except for $\pi$ itself.

Let $N_{n}$ be the number of $B_{n}$-partitions without zero-block. Taking $\pi=\hat{0}^{B}$ in (3.2), we obtain that

$$
N_{n}=\frac{1}{\sqrt{e}} \sum_{k \geqslant 0} \frac{(2 k)^{n}}{(2 k) ! !} .
$$

Let $N_{n}(k)$ denote the number of $B_{n}$-partitions containing $k$ block pairs but no zero-block. It should be noted that the formula (3.3) can be easily deduced from the relation

$$
N_{n}(k)=2^{n-k} S(n, k)
$$

where $S(n, k)$ are the Stirling numbers of the second kind, and the following identity on the Bell polynomials $[9,10]$ :

$$
\sum_{k=0}^{n} S(n, k) x^{k}=\frac{1}{e^{x}} \sum_{k \geqslant 0} \frac{k^{n}}{k !} x^{k} .
$$

Inspecting the proof of Corollary 2.4, we obtain the following result. Let $N_{n, 2}^{D}(k)$ denote the number of ordered pairs $\left(\pi, \pi^{\prime}\right)$ of minimally intersecting $B_{n}$-partitions without zero-block such that $\pi$ consists of exactly $k$ block pairs. Then

$$
N_{n, 2}^{D}(k)=\frac{(2 n) ! !}{(2 k) ! ! \sqrt{e}}\left[x^{n}\right] \sum_{j \geqslant 0} \frac{1}{(2 j) ! !}\left[(1+x)^{2 j}-1\right]^{k} .
$$


The number $N_{n, 2}^{D}$ of ordered pairs $\left(\pi, \pi^{\prime}\right)$ of minimally intersecting $B_{n}$-partitions without zero-block is given by

$$
N_{n, 2}^{D}=\frac{2^{n}}{e} \sum_{k, l \geqslant 0} \frac{(2 k l)_{n}}{(2 k) ! !(2 l) ! !} .
$$

For example, $N_{1,2}^{D}=1, N_{2,2}^{D}=7, N_{3,2}^{D}=75$.

The following theorem is an analogue of Theorem 1.1 with respect to the meetsemilattice of $B_{n}$-partitions without zero-block.

Theorem 3.1 For $r \geqslant 2$, the number of minimally intersecting $r$-tuples $\left(\pi_{1}, \pi_{2}, \ldots, \pi_{r}\right)$ of $B_{n}$-partitions without zero-block equals

$$
N_{n, r}^{D}=\frac{2^{n}}{e^{r / 2}} \sum_{k_{1}, \ldots, k_{r} \geqslant 0} \frac{\left(2^{r-1} k_{1} k_{2} \cdots k_{r}\right)_{n}}{\left(2 k_{1}\right) ! !\left(2 k_{2}\right) ! ! \cdots\left(2 k_{r}\right) ! !} .
$$

Proof. Let $1 \leqslant t \leqslant r$. Let $\mathbf{j}_{t}=\left(j_{t, 1}, j_{t, 2}, \ldots, j_{t, k_{t}}\right)$ be a composition of $n$. Assume that $\pi_{t}$ is of type $\left(0 ; \mathbf{j}_{t}\right)$. Let $N^{D}\left(\pi_{1}, \mathbf{j}_{2}, \ldots, \mathbf{j}_{r}\right)$ be the number of $(r-1)$-tuples $\left(\pi_{2}, \ldots, \pi_{r}\right)$ of such $B_{n}$-partitions such that $\left(\pi_{1}, \pi_{2}, \ldots, \pi_{r}\right)$ is minimally intersecting. By the argument in the proof of Theorem 2.1, we find

$$
N^{D}\left(\pi_{1}, \mathbf{j}_{2}, \ldots, \mathbf{j}_{r}\right)=c \cdot\left[\mathbf{x}^{\mathbf{j}_{1}}\right] \sum_{\mathbf{b}_{s}+\mathbf{c}_{s}=\mathbf{j}_{s}}\left[\mathbf{y}_{2}^{\mathbf{b}_{2}} \overline{\mathbf{y}}_{2}^{\mathbf{c}_{2}} \cdots \mathbf{y}_{r}^{\mathbf{b}_{r}} \overline{\mathbf{y}}_{r}^{\mathbf{c}_{r}}\right] f(\mathbf{j}),
$$

where

$$
f(\mathbf{j})=\prod_{Y_{s} \in\left\{y_{s, 1}, \bar{y}_{s, 1}, \ldots, y_{s, k_{s}}, \bar{y}_{s, k_{s}}\right\}}\left(1+x_{\alpha} Y_{2} Y_{3} \cdots Y_{r}\right) .
$$

Let $N^{D}\left(\pi_{1}, k_{2}, \ldots, k_{r}\right)$ be the number of $(r-1)$-tuples $\left(\pi_{2}, \ldots, \pi_{r}\right)$ of $B_{n}$-partitions such that $\pi_{s}$ consists of $k_{s}$ block pairs, and $\pi_{1}, \pi_{2}, \ldots, \pi_{r}$ are minimally intersecting. It follows from (3.8) that

$$
\begin{aligned}
N^{D}\left(\pi_{1}, k_{2}, \ldots, k_{r}\right) & =c \cdot\left[\mathbf{x}^{\mathbf{j}_{1}}\right] \sum_{\mathbf{b}_{s}+\mathbf{c}_{s}=\mathbf{j}_{s} \geqslant \mathbf{1}}\left[\mathbf{y}_{2}^{\mathbf{b}_{2}} \cdots \overline{\mathbf{y}}_{r}^{\mathbf{c}_{r}}\right] f(\mathbf{j}) \\
& =\mathbf{j}_{1} ! \sum_{l_{2}, \ldots, l_{r}}\left(\left[\mathbf{x}^{\mathbf{j}_{1}}\right] \prod_{\alpha \in\left[k_{1}\right]}\left(1+x_{\alpha}\right)^{2^{r-1} l_{2} \cdots l_{r}}\right) \prod_{2 \leqslant s \leqslant r}\left(\begin{array}{c}
k_{s} \\
l_{s}
\end{array}\right) \frac{(-1)^{k_{s}-l_{s}}}{\left(2 k_{s}\right) ! !} .
\end{aligned}
$$

Consequently,

$$
\begin{aligned}
N_{n, r}^{D} & =\sum_{k_{1}} \frac{1}{\left(2 k_{1}\right) ! !} \sum_{\substack{j_{1,1}+\cdots+j_{1}, k_{1}=n \\
j_{1,1}, \ldots, j_{1, k_{1}} \geqslant 1}} \frac{2^{n} n !}{\mathbf{j}_{1} !} \sum_{k_{2}, \ldots, k_{r}} N^{D}\left(\pi_{1}, k_{2}, \ldots, k_{r}\right) \\
& =(2 n) ! ! \sum_{\substack{k_{1}, k_{2}, \ldots, k_{r} \\
l_{1}, l_{2}, \ldots, l_{r}}} \prod_{1 \leqslant s \leqslant r}\left(\begin{array}{c}
k_{s} \\
l_{s}
\end{array}\right) \frac{(-1)^{k_{s}-l_{s}}}{\left(2 k_{s}\right) ! !}\left[x^{n}\right](1+x)^{2^{r-1} l_{1} l_{2} \cdots l_{r}} .
\end{aligned}
$$


Applying (2.26), we can restate the above formula in the form of (3.7). This completes the proof.

For example, when $n=2$ and $r=3$, by (3.7) we find that $N_{2,3}^{D}=25$. In fact, there are $3 B_{2}$-partitions without zero-block, that is,

$$
0^{B}, \pi_{1}=\{ \pm\{1,2\}\}, \pi_{2}=\{ \pm\{1,-2\}\}
$$

Among all 27 -tuples of $B_{2}$-partitions without zero-block, there are only two partitions $\left(\pi_{1}, \pi_{1}, \pi_{1}\right)$ and $\left(\pi_{2}, \pi_{2}, \pi_{2}\right)$ that are not minimally intersecting.

Acknowledgments. We are grateful to the referee for helpful comments. This work was supported by the 973 Project, the PCSIRT Project of the Ministry of Education, and the National Science Foundation of China.

\section{References}

[1] M. Benoumhani, On Whitney numbers of Dowling lattices, Discrete Math. 159 (1996), 13-33.

[2] A. Björner and F. Brenti, Combinatorics of Coxeter Groups, 2005, Springer Science+Business Media, Inc.

[3] A. Björner and M.L. Wachs, Geometrically constructed bases for homology of partitions lattices of types $A, B$ and D, Electron. J. Combin. 11 (2004), \#R3.

[4] E.R. Canfield, Meet and join within the lattice of set partitions, Electron. J. Combin. 8 (2001), \#R15.

[5] T.A. Dowling, A class of geometric lattices based on finite groups, J. Combin. Theory Ser. B 14 (1973), 61-86.

[6] J.E. Humphreys, Reflection Groups and Coxeter Groups, Cambridge Studies in Advanced Mathematics 29, Cambridge Univ. Press, Cambridge, 1990.

[7] B. Pittel, Where the typical set partitions meet and join, Electron. J. Combin. 7 (2000), \#R5.

[8] V. Reiner, Non-crossing partitions for classical reflection groups, Discrete Math. 177 (1997), 195-222.

[9] J. Riordan, An Introduction to Combinatorial Analysis, Wiley, New York, 1980.

[10] S. Roman, The Umbral Calculus, Academic Press, New York, 1984. 